\title{
Control of post-operative pain and rehabilitation compliance of patients undergoing knee replacement
}

\begin{abstract}
Objective: A correct approach to the rehabilitation of a patient must include an appropriate pain treatment which considers the patient, their history and their needs; of the central analgesics, tapentadol possesses an exclusive MOR-NRI mechanism of action, with an improved efficacy and tolerability profile compared to traditional opioids. The aim of our work was to verify the extent to which pain conditions functional recovery in patients undergoing knee replacement and admitted for specialist rehabilitation after the orthopaedic surgery.

Methods: The open study assessed the analgesia and tolerability of tapentadol PR (50 mg to $150 \mathrm{mg}$ bid) compared to standard treatment (paracetamol $1000 \mathrm{mg}$ bid), in patients in rehabilitation after knee replacement surgery with moderate to severe pain (baseline NRS $\geq 5$ ) The observation lasted 3 weeks, during which there were 4 controls: baseline and after 7, 14 and 21 days. In addition to the dosage of the analgesics being assessed, the following parameters were considered: pain intensity (NRS 0-10), sleep quality (4-point scale), functional recovery (active/passive ROM), muscle tone, Barthel index, comorbidities (CIRS scale) and resilience. Any side effects were also recorded.

Results: 144 patients (104 F/40 M, 44-84 years) were admitted: 91 received tapentadol PR and 53 paracetamol. At the baseline, the 2 groups were found to be homogeneous. During the study, more favourable progress was observed with tapentadol PR: in particular, pain, ROM and sleep quality showed statistically significant and faster improvement in the patients treated with tapentadol PR $(P<0.01)$. At the end of the study, the pain intensity had fallen 4.3 points on the NRS scale $(83 \%)$ with tapentadol PR, compared to $2.4(48 \%)$ with paracetamol. Both drugs were well tolerated.
\end{abstract}

Conclusion: So, for the patient in rehabilitation, tapentadol PR can represent a manageable analgesic that can control the pain, allowing the rehabilitation plan to be carried out.

\section{KEYWORDS: Pain, rehabilitation, knee replacement surgery, resilience}

\section{Introduction}

Post-operative pain after orthopaedic surgery is acute pain that is often intense, and presents complexities linked to different factors, including age, pre-existing conditions, affective integration (anxiety and/or depression) anaesthetic techniques and location and duration of the intervention. It has been shown definitively that this pain must be treated as a true "pathogenic entity" in the post-operative period, and that the alterations it induces can have a significant impact on the clinical progress and outcome of the surgical patient [1]. The literature emphasises the need for a multiintervention strategy to control post-operative pain so as to achieve optimal results and avoid the onset of chronic pain, but to date attention has focussed on the peri-operative period, and there is less knowledge and fewer studies on the rehabilitation period [2,3].

However, pain control continues to be very important in this phase, and a number of considerations may be made, based on the type of intervention and/or the patient. For example, high intensity pain during rehabilitation is significantly associated with a longer stay in hospital, poor compliance with the re-education protocol, delay in resumption of ambulation and an increase in post-operative complications $[4,5]$.

Further considerations arise from the continual ageing of the population, and the evolution in anaesthetic and surgical techniques that allow both faster discharge and operations on patients over eighty or ninety years of age. For all patients, particularly ortho-geriatric ones,a post-operative rehabilitation pathway that enables functional recovery sufficient to retain self-sufficiency and the resumption of daily and social activities is important. Guaranteeing sufficient activity also means maintaining psychological balance and good mood.
Panella $\mathrm{L}^{1 *}$, Caserta $\mathrm{AV}^{2}$, Ballarati $\mathbf{R}^{2}$, Lopresti $\mathbf{M}^{1}$ \& Parravicini $\mathrm{L}^{1}$

${ }^{1}$ Rehabilitation and Functional Recovery, Gaetano Pini Specialist Centre for Orthopaedic Trauma, Milano, Italy

${ }^{2}$ Physical Medicine and Rehabilitation Resident, The University of Milan, Milano, Italy

*Author for correspondence: lorenzo.panella@asst-pini-cto.it 
So a correct approach to the patient in rehabilitation has to include an appropriate pain treatment that considers the patient, their history and their needs, and can thus be considered pharmacologically an "ideal analgesic" to be subjected to clinical evaluation in the various pain conditions.

Of the powerful analgesics, tapentadol is not only the only new molecule, but it also possesses an original mechanism of action: it is a $\mu$ opioid receptor agonist (MOR) and a noradrenaline reuptake inhibitor (NRI); both mechanism of action make complementary and synergic contributions, and its analgesic efficacy has been demonstrated in various pain models, both nociceptive and neuropathic [6,7].

The efficacy of tapentadol in patients with musculo-skeletal pain of various types is the same as that of the strong opioids, from which it differs in having a better tolerability profile, which results in a notably lower risk of abandoning the treatment and a better quality of life for the patient [8-10]. In addition, tapentadol may be administered to elderly patients, and is very safe, due to the low risk of pharmacological interactions due to the reducing binding to plasma proteins, the lack of impact on the CYP450 enzymes (the principal metabolic route in glucuronidation) and the absence of active metabolites [7-11].

The aim of our work was to verify the extent to which pain control affects functional recovery in patients undergoing knee replacement and admitted for specialist rehabilitation after the orthopaedic surgery.

A further aspect that we wanted to consider in our patients was their resilience, i.e. their capacity to deal with events and overcome them, people's capacity to react in the face of adversity [12-14].

Resilience is a term derived from materials science, and means the property that some materials have of conserving their structure or reacquiring their original shape after having been subject to compression or deformation. In psychology it means a person's capacity to face stressful or traumatic events, and reorganise their life in a positive way in the face of difficulties. People with a high level of resilience manage to cope effectively with setbacks, to give new enthusiasm to their existence and even achieve major goals. Exposure to adversity seems to strengthen rather than weaken them; tendentially they are optimistic, flexible and creative; they can work in teams and easily learn from their own and other people's experiences. Five factors are thought to determine resilience: confidence, independence, industriousness, initiative and identity. In determining the resilient capacities of an elderly person, it needs to be remembered that resilience is an entirely personal path; each individual reacts differently to a traumatic event, and help that is valid for one elderly person may not be effective in another. It must also be borne in mind that sometimes an elderly person who is not very resilient may latently retain some energies that must be recognised and activated by those caring for them. These personal resources that are associated in various ways with resilience, should be sought, especially when an elderly person has to face new and stressful emergencies, such as an operation, trauma or the worsening of a chronic-degenerative condition [15].

\section{Materials and Methods}

The study was carried out during 2015 in the Medicina Fisica e Riabilitazione Centro Specialistico Ortopedico Traumatologico Gaetano Pini - Milan.

The experimental design was an open one, to evaluate the analgesic efficacy and tolerability of tapentadol PR compared to the standard treatment with paracetamol in patients undergoing rehabilitation after a knee replacement operation.

Adult patients of both sexes who had undergone knee replacement surgery, with moderate to severe pain (baseline NRS $\geq$ to 5) were admitted to the study. Conversely, patients with oncological pain, contra-indications to the use of tapentadol, patients under 18 years of age and pregnant or breast-feeding women were not included in the study.

Tapentadol PR was administered according to the therapeutic indications, posology and warnings for use listed in the Summary of Product Characteristics; in particular the patients were treated with tapentadol PR, starting treatment when transferred to the rehabilitation specialist, at a dosage of $50 \mathrm{mg}$ bid; if necessary the dosage could be increased by $50 \mathrm{mg}$ bid up to a total daily dose of no more than $500 \mathrm{mg}$ of tapentadol.

A retrospective control group was also identified, homogeneous for demographic and clinical characteristics, treated with $1000 \mathrm{mg}$ of paracetamol tid. 
The observation period lasted 3 weeks, and the data were recorded at the following times: at the baseline (start of analgesic treatment) and after 7, 14 and 21 days, according to management practice of patients in rehabilitation after knee replacement surgery.

In addition to the dosage of analgesic, the following parameters were extrapolated from the patients' medical records: pain intensity assessed on a numerical scale (NRS) from 0 to 10 , sleep quality measured on a 4 -point scale ( $4=$ restorative, 3=good, 2=with frequent awakenings, 1=very disturbed), functional recovery through Range of Motion (active and passive), muscle tone, Barthel index, comorbidities (CIRS scale) and resilience, i.e. the capacity of an individual to face stressful events and overcome them.

In addition, any side effects were recorded (including constipation, nausea, vomiting and headache), describing their severity.

For the statistical analysis, we proceeded as follows: the type variables

- All the variables recorded in the data collection form are reported in descriptive tables. In particular, the continuous variables are presented as means, SD, minimum and maximum, while the discrete and nominal variables are reported in contingency tables such as number and percentage values.

- The range of motion variable (see as angle of total variation) was assessed using a repeated measures ANOVA.

- The muscle tone variable and the Barthel index were evaluated with a $\mathrm{T}$ test for paired data, since they were measured at the start and end of treatment only.

- The pain intensity variable was evaluated through analysis of variance for repeated measures and through a $\mathrm{T}$ test for paired data, on the baseline value and the last value recorded during the study. We chose to use both approaches, given the high number of patients discharged from the unit before the observation period was complete.

- The sleep quality variable was analysed with the McNemar test extended to nxn contingency tables (Bowker test).

- The threshold value used to evaluate the significance of the statistical tests was $0.05(5 \%)$.

- The software used for the analysis was SAS 9.3, while the figures were produced using Microsoft Excel 2013.

\section{Results \\ - Population}

A total of 244 patients $(104 \mathrm{~F}$ and $140 \mathrm{M})$, between 44 and 84 years of age (71.2 \pm 7.7 years, mean \pm SD), with a body weight between 52 $\mathrm{kg}$ and $101 \mathrm{~kg}(72.1 \mathrm{~kg} \pm 9.3 \mathrm{~kg})$, undergoing rehabilitation after knee replacement surgery were admitted to the study.

Ninety-one patients received tapentadol PR and 53 received paracetamol; the 2 treatment groups were homogeneous in terms of their demographic characteristics (TABLE 1).

Regarding the pain characteristics, the mean intensity at the baseline was $5.4( \pm 1.3)$ in the tapentadol PR group and $4.9( \pm 1.9)$ in the paracetamol group. In both groups, most patients presented spontaneous pain at rest (72\%) and/or evoked by movement (56\%), with a continuous type pattern (67\%, TABLE 2$)$.

$30 \%$ of the patients, all in the tapentadol group, had been treated previously with an analgesic, generally paracetamol alone or in association with NSAIDs; this treatment was almost always judged to have poor efficacy and excellent tolerability (over $80 \%$ of the patients).

\section{Treatments}

Upon admission, tapentadol PR was

\begin{tabular}{|c|c|c|c|c|}
\hline & Tapentadol & Paracetamol & Total & $\begin{array}{c}\text { Test of } \\
\text { homogeneity }\end{array}$ \\
\hline Age (years, mean \pm SD) & $71.3 \pm 7.4$ & $70.9 \pm 8.3$ & $71.2 \pm 7.7$ & NS \\
\hline Weight $(\mathrm{kg}$, mean $\pm \mathrm{SD})$ & $72.1 \pm 9.2$ & $71.9 \pm 9.7$ & $72.1 \pm 9.3$ & NS \\
\hline \multicolumn{5}{|l|}{ Diagnosis (No. patients and \%) } \\
\hline - Total replacement right knee joint & $42(46.2)$ & $25(47.2)$ & $67(46.5)$ & \\
\hline - Total replacement left knee joint & $39(42.8)$ & $24(45.3)$ & $63(43.7)$ & \\
\hline $\begin{array}{l}\text { - Replacement of one component of } \\
\text { right knee joint }\end{array}$ & $5(5.5)$ & $1(1.9)$ & $6(4.2)$ & \\
\hline $\begin{array}{l}\text { - Replacement of one component of } \\
\text { left knee joint }\end{array}$ & $1(1.1)$ & $3(5.7)$ & $4(2.8)$ & \\
\hline $\begin{array}{l}\text { - Revision of total replacement of } \\
\text { right knee joint }\end{array}$ & $2(2.2)$ & 0 & $2(1.4)$ & \\
\hline - Revision of tibial component & $1(1.1)$ & 0 & $1(0.7)$ & \\
\hline Tibio-peroneal osteotomy & $1(1.1)$ & 0 & $1(0.7)$ & \\
\hline \multicolumn{5}{|l|}{ CIRS } \\
\hline - Severity $(m \pm S D)$ & $1.41 \pm 0.16$ & $1.40 \pm 0.17$ & $1.40 \pm 0.16$ & NS \\
\hline \multicolumn{5}{|l|}{ - Comorbidities (No. patients and \%) } \\
\hline 1 & $47(51.6)$ & $23(43.4)$ & $70(48.6)$ & \\
\hline 2 & $30(33)$ & $20(37.7)$ & $50(34.79$ & \\
\hline 3 & $10(11)$ & $8(15.1)$ & $18(12.5)$ & \\
\hline 4 & $2(2.2)$ & $2(3.8)$ & $4(2.8)$ & \\
\hline 5 & $2(2.2)$ & 0 & $2(1.4)$ & \\
\hline Barthel Index $(m \pm S D)$ & $80.0 \pm 9.2$ & $76.1 \pm 11.6$ & & NS \\
\hline Resilience $(\mathrm{m} \pm \mathrm{SD})$ & $140.0 \pm 22.9$ & $140.9 \pm 22.4$ & $140.4 \pm 22.5$ & NS \\
\hline
\end{tabular}




\section{Table 2. Pain characteristics on admission.}

\begin{tabular}{|l|c|c|c|}
\hline & Tapentadol & Paracetamol & Total \\
\hline Pain (No. patients and \%) & & & \\
\hline - Spontaneous at rest & $68(74.7)$ & $35(66)$ & $103(71.5)$ \\
\hline - Evoked by load & $32(35.2)$ & $10(18.9)$ & $42(29.2)$ \\
\hline - Evoked by movement & $47(51.6)$ & $33(62.3)$ & $80(55.6)$ \\
\hline Pain (No. patients and \%) & & & \\
\hline - Continuous & $57(73.6)$ & $30(56.6)$ & $97(67.4)$ \\
\hline - Intermittent & $20(22)$ & $13(24.5)$ & $33(22.9)$ \\
\hline - Episodic & $3(3.3)$ & $10(18.9)$ & $13(9)$ \\
\hline Intermittent/episodic & $1(1.1)$ & 0 & $1(0.7)$ \\
\hline
\end{tabular}

prescribed at the dosage of $50 \mathrm{mg}$ bid in 66 of the 91 patients (equivalent to $73 \%$; in $50 \%$ of cases alone and in 50\% in association with NSAIDs), and at the dosage of $100 \mathrm{mg}$ bid in 25 of the 91 patients (equivalent to $27 \%$, in $25 \%$ of cases alone and in $75 \%$ in association with NSAIDs). While in the paracetamol group the initial dosage was always $1000 \mathrm{mg}$ bid (in $92 \%$ of the cases, on its own, and in $8 \%$ in association with NSAIDs).

Over the course of the study, 43 of the patients in the tapentadol PR group did not change the initial dosage ( 35 receiving $50 \mathrm{mg}$ bid and 8 receiving $100 \mathrm{mg}$ bid), 18 patients just increased it (11 receiving $50 \mathrm{mg}$ bid and 7 receiving $100 \mathrm{mg}$ bid; maximum dosage: 150 mg bid), 9 just reduced it (all receiving 100 $\mathrm{mg}$ bid) and in 21 patients the initial dose was modified, both increasing and decreasing it (20 receiving $50 \mathrm{mg}$ bid and 1 receiving $100 \mathrm{bid}$ ).

In total, 16 patients suspended the treatment with tapentadol PR: in 5 cases due to recovery/ discharge, in 7 cases due to adverse effects, in 2 cases at the request of the patient and in another 2 cases the reason was not specified.

\section{- Analgesic efficacy}

Over the course of the study, a reduction in pain intensity was observed in both treatment groups: with tapentadol PR the mean value changed from 5.2 points at the baseline to 0.9 points after 3 weeks of treatment $(-4.3$, a reduction of $83 \%$ ), while with paracetamol the mean value changed from 5.0 points to 2.6 points after 3 weeks of treatment $(-2.4$, a reduction of $48 \%$, FIGURE 1); the difference between the two treatments was statistically significant $(\mathrm{P}<0.01)$.

It should also be noted that there was a faster response in the tapentadol PR group, where there was a more substantial reduction in the first week, while for paracetamol the reduction was more substantial in the third week.

\section{Sleep quality}

There was a progressive and marked improvement in sleep quality; in particular, there was a fall in the percentage of "very disturbed" responses and an increase in the percentage of "good" responses. At the end of the observation period, the percentage of "good" responses was $68 \%$ in the tapentadol PR group and $46 \%$ in the paracetamol group. The comparison of the two groups was statistically significant after two $(\mathrm{P}<0.05)$ and three $(\mathrm{P}<0.01)$ weeks of treatment (FIGURE 2).

\section{- Joint function}

The range of motion of the knee joint showed a clear improvement over the course of the

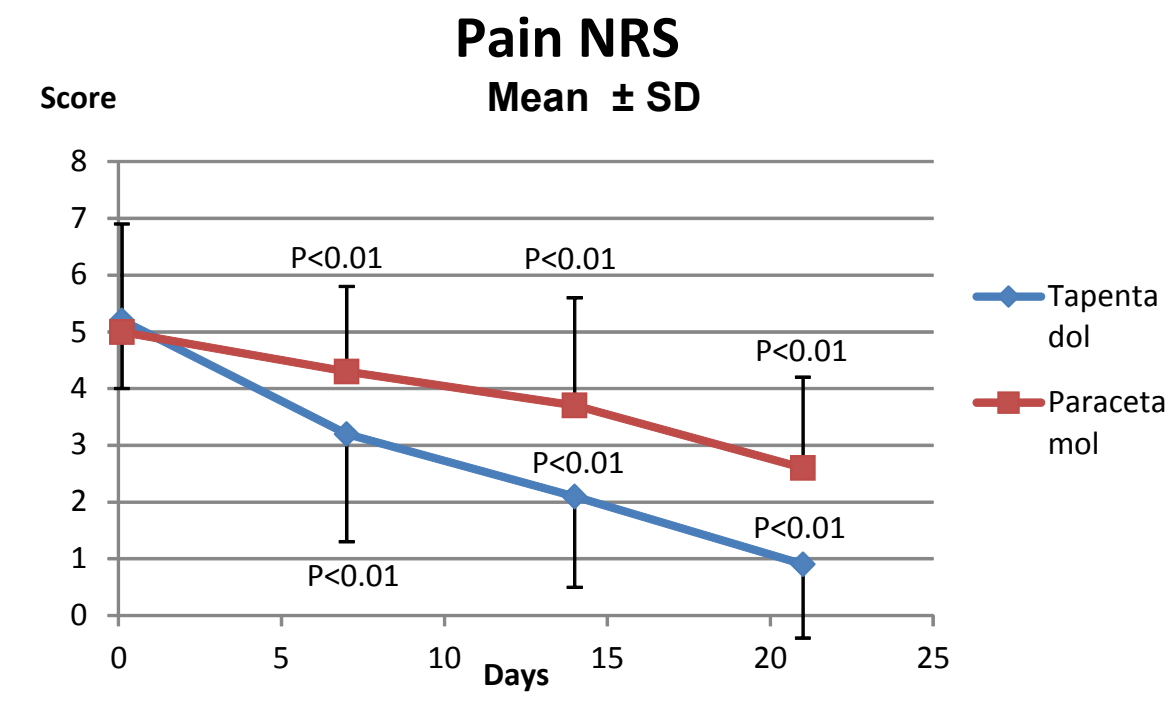

Figure 1. Pain intensity during the 3 weeks of the study in patients receiving tapentadol PR or paracetamol. 
observation. In the subjects receiving tapentadol PR, there was a mean overall increase of $118 \%$ in active ROM (FIGURE 3A) and $87 \%$ in passive ROM (FIGURE 3B), while in the paracetamol group the mean overall increase was $50 \%$ in passive $\mathrm{ROM}$ and $74 \%$ in active $\mathrm{ROM}$ (FIGURE 1). The difference between the two treatments was statistically significant $(\mathrm{P}<0.01)$.

\section{- Muscle tone}

In the 3 weeks of observation, there was an improvement, and, specifically, the mean muscle tone value increased by $37 \%$ in the tapentadol PR group and 34\% in the paracetamol group (NS between the groups).

\section{- Activities of daily life}

The Barthel index, which already indicated a high degree of autonomy at the baseline, improved over the 3 weeks of the study, reaching total autonomy. In the tapentadol PR group it changed from a mean of 80 at the baseline to a final value of 97, and in the paracetamol group from a mean of 76 at the baseline to a final value of 95 (NS between the groups).

\section{- Resilience}

No correlation was found between pain intensity and resilience (correlation coefficient $0.054, \mathrm{NS})$.
To define the logistic regression model, all those patients who presented a pain intensity score $\leq 3$ NRS were considered responders. The model was estimated using a step-wise selection procedure, that is, considering only the treatment carried out, paracetamol or tapentadol PR; the value for the OR between tapentadol PR and paracetamol obtained was 0.120 ; this means that the patients treated with paracetamol had a probability of being responders of less than $88 \%$, compared to the patients treated with tapentadol PR; this difference in probability was statistically significant $(\mathrm{P}<0.01)$.

\section{Tolerability}

The tolerability was good in both treatment groups; in particular, $41 \%$ of the patients (a total of 60 events) in the group treated with tapentadol PR, and $36 \%$ of the patients (a total of 27 events) in the group treated with paracetamol reported at least one adverse event; in both treatment groups the most frequent side effect was constipation. In general, the symptoms were always mild, apart from one case of moderate nausea reported with tapentadol PR.

\section{Discussion}

Treating pain is fundamental not only for ethical reasons but also because in rehabilitation this facilitates joint recovery after orthopaedic

\section{Sleep Quality}

Very disturbed With frequent awakenings Good a Restorative

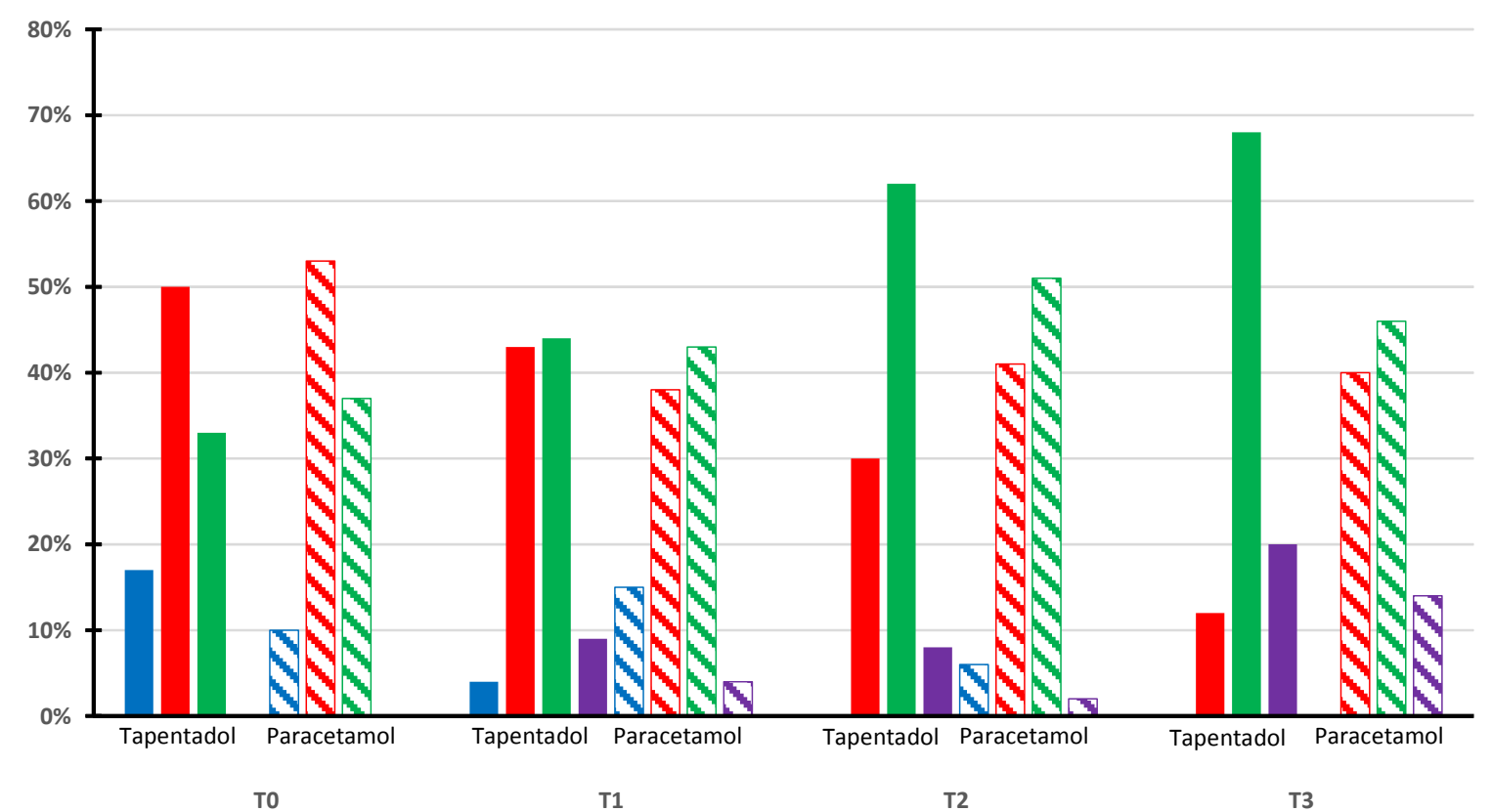

Figure 2. Sleep quality during the 3 weeks of the study in patients receiving tapentadol PR or paracetamol. 
A

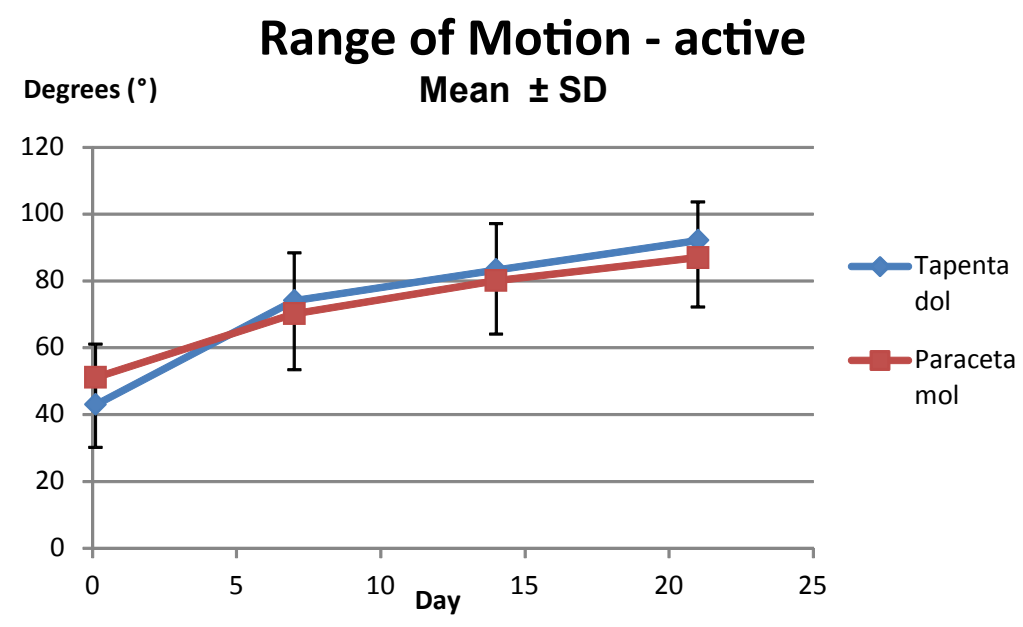

B

\section{Range of Motion - passive Degrees $\left({ }^{\circ}\right) \quad$ Mean \pm SD}

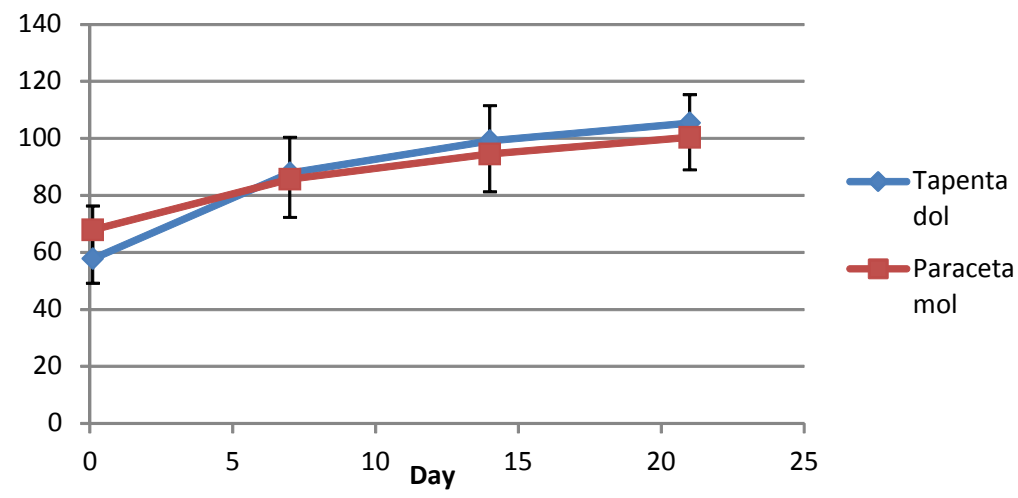

Figure 3. Joint function (A: active Range of Motion; B: passive Range of Motion) of the knee joint during the 3 weeks of the study in patients receiving tapentadol PR or paracetamol. of assessing the efficacy and tolerability of tapentadol PR in a group of patients who had undergone knee replacement surgery, compared to the standard treatment with paracetamol. As was to be expected, the results show a progressive improvement in all the parameters over the course of the rehabilitation. However, the analgesic result and functional recovery of the knee joint were significantly faster and better in the patients treated with tapentadol PR. Sleep quality, which is important for the well-being of the patient, also improved in a significantly faster and more marked way in the tapentadol PR group.

It should be emphasised that in addition to the therapeutic result, tolerability was also good, insofar as no serious or severe adverse events occurred.

The better results obtained in the patients treated with tapentadol PR may be ascribed to its better coverage of all the pain components, nociceptive and neuropathic, which is guaranteed by this molecule, characterised by a dual MOR-NRI mechanism of action, unique among all the analgesics currently available $[6,7]$.

Furthermore, at spinal cord level, tapentadol combats the process of pain chronicisation, reducing ascending pain inputs and strengthening descending inhibitory control through the noradrenergic pathways. This activity may be particularly advantageous in patients with recurring or persistent pain evolving towards chronicity $[7,16]$.

A typical example of recurrent pain is arthrosic reacutisation, in which painful episodes become relatively frequent (monthly) and relatively unresponsive to non-steroidal antiinflammatories, the painful area extends and paraesthesias can appear. In the post-operative patient, the pain can, if not adequately treated, become a persistent pain, i.e., a pain that lasts for more than 3 months and is not fully resolved with the healing of the peripheral trauma. In orthopaedics and in rehabilitation, painful joint replacements are by now relatively common: after total knee replacement $44 \%$ of patients present persistent pain that in $13 \%$ of cases has features typical of neuropathic pain [17].

In all these situations, tapentadol is an appropriate drug, because it can rebalance the physiological pain control, preventing or slowing the evolution of the pain towards chronicity. 
As for the assessment of resilience, no correlation with the pain intensity was found in the patients assessed in this study, but the differing responses to the two treatments was confirmed, resulting in a probability of optimal pain control $(\mathrm{NRS} \leq 3)$ that is clearly higher in

\section{REFERENCES}

Meissner W, Coluzzi F, Fletcher D et al. Improving the management of post-operative acute pain: priorities for change. Curr. Med. Res. Opin. 31(11), 2131-2143 (2015).

Pergolizzi JV, Raffa RB, Taylor R. Treating acute pain in light of the chronification of pain. Pain Manag. Nurs. 15(1), 380-390 (2014).

Gerbershagen HJ. Transition from acute to chronic postsurgical pain. Physiology, risk factors and prevention. Schmerz 27(1), 81-93 (2013)

Murena L, Cherubino P, Ratti C. La gestione del dolore persistente in ortopedia. Minerva Ortopedica e Traumatologica 63(6), 1-7 (2012).

Scardino M, Intelligent F. The musculoskeletal pain and current analgesic options. (2) SIGASCOT (2014).

Tzschentke TM. Tapentadol hydrochloride: a next generation, centrally acting analgesic with two mechanisms of action in a single molecule. Drugs Today (Barc).45(7), 483-496 (2009).

Canonico PL, Grilli M, Fornasari D, Romualdi P, Sacerdote P. Tapentadolo: l'innovazione

the group treated with tapentadol PR compared to the group receiving paracetamol.

So for the patient in rehabilitation, tapentadol PR can represent a manageable analgesic that can control the pain, allowing the rehabilitation plan to be carried out.

farmacologica per il dolore. Minerva Medica. 105, 1-31 (2014).

Baron R, Likar R, Martin-Mola E et al. Effectiveness of Tapentadol Prolonged Release (PR) Compared with Oxycodone/Naloxone PR for the Management of Severe Chronic Low Back Pain with a Neuropathic Component: A Randomized, Controlled, Open-Label, Phase 3b/4 Study. Pain Pract. 16(5), 580-599 (2015).

Baron R, Jansen JP, Binder A et al. Tolerability, Safety, and Quality of Life with Tapentadol Prolonged Release (PR) Compared with Oxycodone/Naloxone PR in Patients with Severe Chronic Low Back Pain with a Neuropathic Component: A Randomized, Controlled, Open-Label, Phase 3b/4 Trial. Pain Pract. 16(5), 600-619 (2015).

Biondi DM, Xiang J, Etropolski M, Moskovitz B. Tolerability and efficacy of tapentadol extended release in elderly patients $\geq 75$ years of age with chronic osteoarthritis knee or low back pain. J. Opioid Manag. 11(5), 393-403 (2015).

Buynak R, Rappaport SA, Rod K et al. Long-term Safety and Efficacy of Tapentadol Extended
Release Following up to 2 Years of Treatment in Patients With Moderate to Severe, Chronic Pain: Results of an Open-label Extension Trial. Clin. Ther. 37(11), 2420-2438 (2015).

Development and psychometric evaluation of the Resilience Scale. J. Nurs. Meas. 1(2), 165-178 (1993).

Browne CJ. Attachment theory, ageing and dementia: a review of the literature. Aging Ment. Health 10(2), 134-142 (2006).

Wallerstein, N. "What is the evidence on effectiveness of empowerment to improve health?." (2006).

Thakur M, Dickenson AH, Baron R. Osteoarthritis pain: nociceptive or neuropathic? Nat. Rev. Rheumatol. 10(6), 374-380 (2014).

Callegari C, Bertu L, Caselli I, Vender S. Resilience in older adults: influence of the admission in nursing home and psychopathology. Neuropsychiatry 6(4), 117-123 (2016).

Coluzzi F e Fornasari D. Dall'acuto al cronico: Tapentadolo nelle fasi evolutive della patologia dolore. Fighting Pain 3(1), 1-7 (2016). 